\title{
Effective nucleon mass and thermodynamics in the quark-meson coupling model
}

\author{
Chen Wu, ${ }^{1}$ Yu-Gang Ma, ${ }^{1}$ Sanjeev Kumar, ${ }^{1}$ Xi-Guang Cao, ${ }^{1}$ and Wei-Liang Qian ${ }^{2}$ \\ ${ }^{1}$ Shanghai Institute of Applied Physics, Chinese Academic of Sciences, Shanghai 201800, China \\ ${ }^{2}$ Universidade de Ouro Preto, Ouro Preto, 35400-000, Brazil
}

(Received 19 July 2012; revised manuscript received 26 September 2012; published 14 December 2012)

\begin{abstract}
In this work, we study the quark energy spectrum in an MIT bag using the quark-meson coupling model. In particular, thermal distribution functions of quarks at finite temperature are explicitly incorporated into the calculations. It is found that when temperature is less than $50 \mathrm{MeV}$, the probability for a quark to occupy an exciting state is very rare and negligible, therefore the corresponding contributions to the thermodynamic potential under mean-field theory are strongly suppressed. In this context, if one calculates other physical quantities derived from thermodynamic potential, effects due to quark distribution functions are also likely to be unimportant.
\end{abstract}

DOI: $10.1103 /$ PhysRevC.86.068201

PACS number(s): 12.39.Ba, 24.85.+p, 21.65.-f

Quark- meson coupling (QMC) is a hybrid model which has successfully described many physical properties of nuclear matter and nuclei [1-3]. In this model, the nuclear system is described as a collection of non-overlapping MIT bags in which quarks interact through the exchange of scalar and vector mesons. The interactions between quarks and mesons are limited within the MIT bag region.

Song and $\mathrm{Su}$ [4] extended the QMC model to include the effect of finite temperature. In their calculations, they incorporated the thermal distribution functions of nucleons and antinucleons when evaluating the energy and the pressure of the system. They found that the equation of state (EOS) at finite temperature is much softer than that in quantum hadron dynamics (QHD) and it is comparable to that in the Zimanyi-Moszkowski model. Furthermore, they noted that the critical temperature of the liquid-gas phase transition (LGPT) is lower in the QMC when compared to that in QHD.

Panda et al. [5] have also studied hot nuclear matter within the framework of the QMC model. In their calculations, however, not only are the thermal distribution functions of nucleons considered but also those of single-particle quarks and antiquarks are considered. The energy of a nucleon bag at finite temperature $T$ was evaluated by summing up all the energy levels of quarks and antiquarks according to the Fermi-Dirac distribution. The resulting nucleon mass at finite temperature was found to be appreciably different from that in a vacuum. This approach has been applied to various nuclear and stellar problems, for example, the study of LGPTs [6], protoneutron stars [7], rotating neutron stars in the Komatsu-Eriguchi-Hachisu method [8], and hybrid stars, which consist of both hadrons and quark matter [9].

In this work, we address the key features of the QMC model at finite temperature [5]. The numerical results of two different methods are presented and discussed.

In the QMC model, the nucleons are described by static MIT bags in which quarks interact with the scalar $(\sigma)$ and the vector $(\omega)$ fields; the latter is usually treated by the mean-field approximation. The quark field $\psi_{q}(\vec{r}, t)$ inside the bag satisfies the Dirac equation:

$$
\left[i \gamma^{\mu} \partial_{\mu}-\left(m_{q}-g_{\sigma}^{q} \sigma\right)-g_{\omega}^{q} \omega \gamma^{0}\right] \psi_{q}(\vec{r}, t)=0,
$$

where $m_{q}^{0}$ is the current quark mass and $g_{\sigma}^{q}$ and $g_{\omega}^{q}$ are the coupling constants between the quark and the $\sigma$ and $\omega$ mesons.

The normalized wave function for a quark in the orbit $(n, \kappa)$ (where $n$ specifies the number of radial nodes and $\kappa$ specifies the angular quantum numbers) of a spherically symmetric MIT bag is given by

$$
\psi_{q}^{n \kappa}(\vec{r}, t)=N e^{-i \epsilon_{q}^{n \kappa} t / R}\left(\begin{array}{c}
j_{l_{k}}(x r / R) \\
i \beta_{q} \operatorname{sgn}(\kappa) \cdot \vec{\sigma} \cdot \hat{r} j_{\bar{l}_{\kappa}}(x r / R)
\end{array}\right) \frac{\chi_{q}}{\sqrt{4 \pi}},
$$

with

$$
\begin{aligned}
\beta_{q} & =\sqrt{\frac{\Omega_{q}^{n \kappa}-R m_{q}^{*}}{\Omega_{q}^{n \kappa}+R m_{q}^{*}}}, \\
\Omega_{q}^{n \kappa} & =\left(x_{n \kappa}^{2}+R^{2} m_{q}^{* 2}\right)^{1 / 2},
\end{aligned}
$$

where $l_{\kappa}=-\kappa-1$ for $\kappa<0$ and $\kappa$ for $\kappa>0 ; \bar{l}_{\kappa}=-\kappa$ for $\kappa<0$ and $\kappa-1$ for $\kappa>0, \operatorname{sgn}(\kappa)=1$ for $\kappa>0$ and -1 for $\kappa<0, N$ is the normalization factor, $R$ is the bag radius, $\chi_{q}$ indicates the quark spinor, and $j_{l}(x r / R)$ is the spherical Bessel function. The single-particle quark energy reads

$$
\epsilon_{q}^{n \kappa}=\frac{\Omega_{q}^{n \kappa}}{R}+g_{\omega}^{q} \omega
$$

where $m_{q}^{*}=m_{q}^{0}-g_{\sigma}^{q} \sigma$ is the effective quark mass.

The boundary condition at the bag surface is given by

$$
i \gamma n \psi_{q}^{n \kappa}=\psi_{q}^{n \kappa} .
$$

By using the explicit form of the above wave function, the boundary condition can be reduced to

$$
j_{|\kappa|-1}(x)+\beta_{q}^{n \kappa} \operatorname{sgn}(\kappa) j_{|\kappa|}(x)=0,
$$

which determines the dimensionless quark momentum $x_{n \kappa}$.

At zero temperature, the three quarks in the MIT bag occupy the ground state with $n=1$ and $\kappa=-1$. At finite temperature, the quarks as well as the antiquarks can be thermally excited to high angular momentum states. The single-particle quark and antiquark energies are given as

$$
\epsilon_{ \pm}^{n \kappa}=\frac{\Omega^{n \kappa}}{R} \pm g_{\omega}^{q} \omega
$$


The total energy from quarks and antiquarks then reads

$$
E_{\mathrm{tot}}=3 \sum_{n, \kappa} \frac{\Omega^{n \kappa}}{R}\left(f_{n \kappa}+\bar{f}_{n \kappa}\right),
$$

where

$$
\begin{aligned}
f_{n \kappa} & =\frac{1}{e^{\left(\epsilon_{+}^{n \kappa} / R-\mu_{q}\right) / T}+1}, \\
\bar{f}_{n \kappa} & =\frac{1}{e^{\left(\epsilon_{-}^{n \kappa} / R+\mu_{q}\right) / T}+1} .
\end{aligned}
$$

Here $\mu_{q}$ is the chemical potential for the quark which is determined through $n_{q}$ as follows:

$$
n_{q}=3=3 \sum_{n, \kappa}\left(f_{n \kappa}-\bar{f}_{n \kappa}\right),
$$

and the resulting bag energy becomes

$$
E_{\text {bag }}=E_{\text {tot }}-\frac{Z}{R}+\frac{4 \pi R^{3} B}{3},
$$

where $Z$ is a parameter accounting for the zero-point energy and $B$ is the bag constant. The entropy of the bag is defined as

$$
\begin{aligned}
S_{\mathrm{bag}}= & -3 \sum_{n, \kappa}\left[f_{n \kappa} \ln f_{n \kappa}+\left(1-f_{n \kappa}\right) \ln \left(1-f_{n \kappa}\right)\right. \\
& \left.+\bar{f}_{n \kappa} \ln \bar{f}_{n \kappa}+\left(1-\bar{f}_{n \kappa}\right) \ln \left(1-\bar{f}_{n \kappa}\right)\right],
\end{aligned}
$$

and the free energy for the bag is given by

$$
F_{\text {bag }}=E_{\text {bag }}-T S_{\text {bag }} \text {. }
$$

In Ref. [5], the nucleon mass is assumed to be the bag energy, $E_{\text {bag. }}$. In our calculations, we take the effective mass of a nucleon bag to be the free energy,

$$
M_{N}^{*}=F_{\text {bag }},
$$

as in Ref. [7]. At zero temperature, both scenarios give the same value for the effective nucleon mass. However, as shown in the following, they lead to opposite behaviors when one studies the nucleon mass and the bag radius as a function of temperature. For reasons that are discussed below, it seems to us to be more desirable to choose free energy as the nucleon mass of the model.

Once the nucleon mass is defined, the bag radius can be obtained through the condition

$$
\frac{\partial M_{N}^{*}}{\partial R}=0
$$

The set of parameters used in the present study is the same as that in Ref. [7]. We take the bag parameters for the free nucleon as $B^{1 / 4}=210.8 \mathrm{MeV}, Z=4.005$, and the current quark mass $m_{q}^{0}=0$. The values of the coupling constants between the mesons and the nucleon as fixed by the saturation properties of the nuclear matter are given as $g_{\sigma}^{q}=5.95$ and $g_{\omega}^{q}=2.99$.

Now we are in a position to present the numerical results. In Table I, the low-lying energy eigenvalues of the quark states in the MIT bag are shown. For $\kappa=-1$ we obtain, for example, the solutions $x_{n,-1} \approx 2.043,5.396,8.578, \ldots$. The ground state corresponds to the orbit $(n=1, \kappa=-1)$. One observes from Table I that the spacing of the energy spectra for a given $\kappa$ is almost a constant.
TABLE I. Energy spectrum $x_{n \kappa}$ in the MIT bag with $\sigma=0$ and $\omega=0$.

\begin{tabular}{lccccc}
\hline \hline & $\kappa=-1$ & $\kappa=1$ & $\kappa=-2$ & $\kappa=2$ & $\kappa=-3$ \\
\hline$n=1$ & 2.043 & 3.811 & 3.203 & 5.123 & 4.327 \\
$n=2$ & 5.396 & 7.002 & 6.757 & 8.407 & 8.059 \\
$n=3$ & 8.577 & 10.16 & 10.00 & 11.61 & 11.37 \\
$n=4$ & 11.73 & 13.31 & 13.19 & 14.78 & 14.60 \\
$n=5$ & 14.88 & 16.46 & 16.37 & 17.95 & 17.80 \\
\hline \hline
\end{tabular}

In Fig. 1, we plot the value of $M_{N}^{*}$ as a function of temperature for the cases $\rho_{B}=0$ and $0.15 \mathrm{fm}^{-3}$. The value of $M_{N}^{*}$ decreases monotonically with temperature. At $\rho_{B}=$ $0 \mathrm{fm}^{-3}$, the effective mass of the nucleon is almost a constant ( $939 \mathrm{MeV})$ for a low-temperature range $(T<50 \mathrm{MeV})$. On the other hand, when $T>50 \mathrm{MeV}$, the effective nucleon mass decreases rapidly with temperature. In Fig. 2, the value of $M_{N}^{*}$ as a function of temperature is shown when the effective nucleon mass is taken to be the bag energy: $M_{N}^{*}=E_{\text {bag. }}$ In contrast to Fig. 1 , it is clearly shown that the effective nucleon mass increases rapidly with temperature. The observed increase of the nucleon mass in Fig. 2 is due to a significant contribution to the bag energy arising from the thermal excitations of the quarks inside the nucleon bag when the temperature is high. On the contrary, when one assumes the free energy to be the effective nucleon mass as shown in Fig. 1, an extra term, namely, $-T S$ in the definition of free energy, becomes dominant when the temperature increases. Consequently, the nucleon mass deceases at high temperature in this case.

For comparison purposes, we also calculated the nucleon radius as a function of temperature for both scenarios. The results are shown in Fig. 3. In both cases, the temperature dependence is not very strong. However, very different behaviors were again observed, namely, the bag radius decreased with

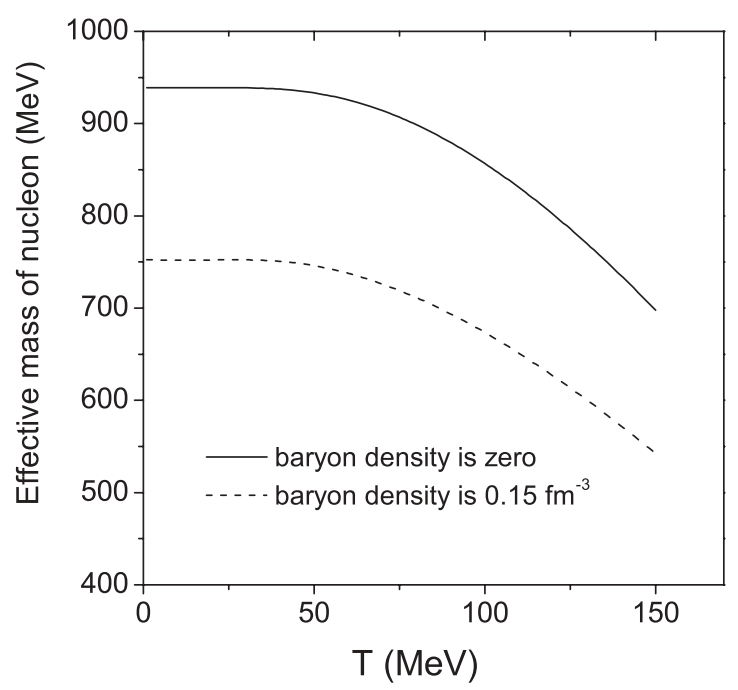

FIG. 1. Effective mass of the nucleon as a function of temperature. The effective nucleon mass is assumed to be the free energy of the MIT bag. The solid line is for the case of $\rho_{B}=0$ and the dashed line is for $\rho_{B}=0.15 \mathrm{fm}^{-3}$. 


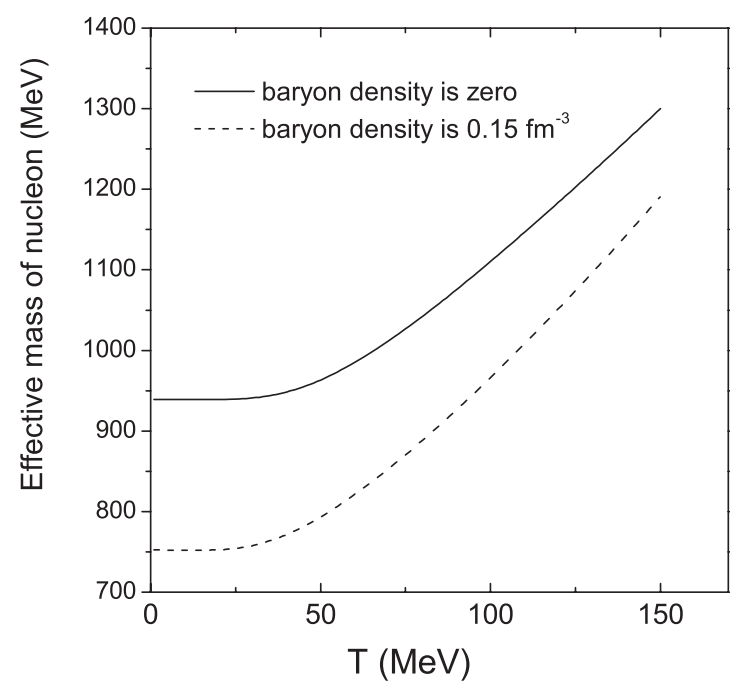

FIG. 2. The same as Fig. 1, but taking the effective mass to be the bag energy.

temperature when adopts the bag energy was adopted and it increased with temperature when the free energy was used. This result motivated us to choose the free energy over the bag energy as the nucleon mass.

We then study the quark and antiquark distributions at different energy levels. In Table II, we enumerate the distribution probabilities of a quark or an antiquark to occupy the low-lying energy levels. For simplicity. we take $\sigma=0$ and $\omega=0$ (i.e., $\left.\rho_{B}=0\right)$. The reason is as follows: it is clear from Eqs. (3)-(10) that the resulting possibility depends on the mean field of the meson fields $\sigma$ and $\omega$; however, the numerical results show that the possibility is actually not very sensitive to the magnitude of the fields. From Table II, one observes that the probability to find a $(u$ or $d$ ) quark in the ground state is around 0.979, while
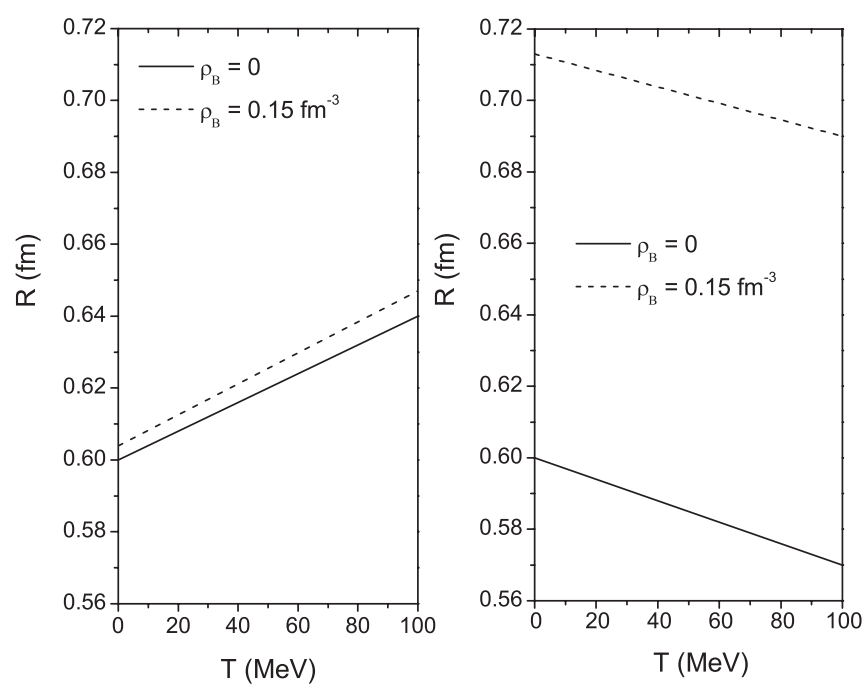

FIG. 3. Radius of the nucleon as a function of temperature. The left panel is obtained when the nucleon mass is chosen to be the free energy; the right panel shows the corresponding results when one uses the bag energy instead.
TABLE II. Quark and antiquark distribution at $T=50 \mathrm{MeV}$ and $\rho_{B}=0$.

\begin{tabular}{lcc}
\hline \hline & Quark & Antiquark \\
\hline$\epsilon^{1,-1}$ & 0.979 & $2.80 \times 10^{-14}$ \\
$\epsilon^{1,-2}$ & $1.99 \times 10^{-2}$ & $1.18 \times 10^{-17}$ \\
$\epsilon^{1,1}$ & $3.50 \times 10^{-14}$ & $2.03 \times 10^{-19}$ \\
$\epsilon^{2,-1}$ & $8.73 \times 10^{-9}$ & $5.08 \times 10^{-24}$ \\
$\epsilon^{2,1}$ & $1.88 \times 10^{-13}$ & $1.09 \times 10^{-28}$ \\
$\epsilon^{2,-2}$ & $9.65 \times 10^{-13}$ & $5.62 \times 10^{-28}$ \\
\hline \hline
\end{tabular}

the occupation probability of the first excited state $(n=1$, $\kappa=-2$ ) is 0.02 , much smaller compared to that of the ground state. Therefore, one concludes that when the temperature is $50 \mathrm{MeV}$ the quark basically only occupies the ground state and the first excited state, and the possibility for a quark to occupy any other excited state is negligible. In particular, at $T=20 \mathrm{MeV}$, a quark can be found in the $1 s$ state with a probability of more than 0.999 . As a result, when one calculates the thermodynamic potential or any quantity derived from it at low temperature, the contributions from excited states are negligible. In Table III, we have shown our results for the quark and antiquark distribution at the different energy levels for $T=100 \mathrm{MeV}$. Once again, we found that the occupation probabilities of quarks in excited states are quite small. The quark, for the most part, occupies only the following states: $\epsilon^{1,-1}, \epsilon^{1,1}, \epsilon^{1,-2}$, and $\epsilon^{1,2}$.

In summary, in this work we presented the analytic solution of quark wave functions and the equation for the quark energy spectrum in an MIT bag model at finite temperature. We studied the probability for quarks to occupy different quantum states $(n, \kappa)$. It is found that the contributions from the ground state and from the first few excited states to the effective nucleon mass are dominant. More precisely, it is observed that for $T \leqslant 50$ the quark stays almost in the ground state. As a consequence, the corresponding contributions to the thermodynamic potential or any quantity derived from it are strongly suppressed. For instance, if one studies the LGPT under the mean-field approximation or the protoneutron stars with the QMC model, such effects due to quark distribution functions are likely to be unimportant. On the other hand, further efforts

TABLE III. Quark and antiquark distribution at $T=100 \mathrm{MeV}$ and $\rho_{B}=0$.

\begin{tabular}{lcc}
\hline \hline & Quark & Antiquark \\
\hline$\epsilon^{1,-1}$ & 0.868 & $1.39 \times 10^{-7}$ \\
$\epsilon^{1,-2}$ & 0.112 & $2.67 \times 10^{-9}$ \\
$\epsilon^{1,1}$ & $1.58 \times 10^{-2}$ & $3.38 \times 10^{-10}$ \\
$\epsilon^{1,2}$ & $2.77 \times 10^{-3}$ & $5.85 \times 10^{-11}$ \\
$\epsilon^{1,-3}$ & $1.85 \times 10^{-4}$ & $3.90 \times 10^{-12}$ \\
$\epsilon^{2,-1}$ & $7.32 \times 10^{-5}$ & $1.54 \times 10^{-12}$ \\
$\epsilon^{2,1}$ & $3.09 \times 10^{-7}$ & $6.53 \times 10^{-15}$ \\
$\epsilon^{2,-2}$ & $7.11 \times 10^{-7}$ & $1.50 \times 10^{-14}$ \\
$\epsilon^{2,2}$ & $8.48 \times 10^{-9}$ & $1.78 \times 10^{-16}$ \\
$\epsilon^{2,-3}$ & $2.59 \times 10^{-9}$ & $5.47 \times 10^{-17}$ \\
\hline \hline
\end{tabular}


should be made as one steps out of the framework of mean-field theory or goes into high-temperature regions.

The authors would like to acknowledge funding from the National Natural Science Foundation of China (NNSFC Grants
No. 11105072 , No. 10979074, No. 10875160, No. 10805067 , and No. 11035009), the Brazilian Foundations Fundação de Amparo á Pesquisa do Estado de Minas Gerais (FAPEMIG) and the Conselho Nacional de Desenvolvimento Cientitífico e Tecnológico (CNPq).
[1] P. A. M. Guichon, Phys. Lett. B 200, 235 (1988).

[2] K. Saito and A. W. Thomas, Phys. Lett. B 327, 9 (1994).

[3] K. Saito, K. Tsushima, and A. W. Thomas, Prog. Part. Nucl. Phys. 58, 1 (2007), and references therein.

[4] H. Q. Song and R. K. Su, Phys. Lett. B 358, 179 (1995).

[5] P. K. Panda, A. Mishra, J. M. Eisenberg, and W. Greiner, Phys. Rev. C 56, 3134 (1997).
[6] P. K. Panda, G. Krein, D. P. Menezes, and C. Providência, Phys. Rev. C 68, 015201 (2003).

[7] P. K. Panda, C. Providência, and D. P. Menezes, Phys. Rev. C 82, 045801 (2010).

[8] P. K. Panda, R. Sahu, and C. Das, Phys. Rev. C 60, 038801 (1999).

[9] P. K. Panda, D. P. Menezes and C. Providência, Phys. Rev. C 69, 025207 (2004); 69, 058801 (2004). 